Internat. J. Math. \& Math. Sci.

Vol. 23, No. 7 (2000) 441-448

S0161171200001587

(C) Hindawi Publishing Corp.

\title{
A GENERAL EXISTENCE PRINCIPLE FOR FIXED POINT THEOREMS IN $D$-METRIC SPACES
}

\author{
B. C. DHAGE, A. M. PATHAN, and B. E. RHOADES
}

(Received 13 April 1998 and in revised form 22 July 1998)

\begin{abstract}
We establish two general principles for fixed point theorems in $D$-metric spaces, and then show that several theorems in $D$-metric spaces follow as corollaries of these general principles.
\end{abstract}

Keywords and phrases. $\alpha$-condensing maps, $D$-metric spaces, fixed point theorems.

2000 Mathematics Subject Classification. Primary 47H10.

1. Introduction. The concept of a $D$-metric space was introduced by the first author in [1]. A nonempty set $X$, together with a function $D: X \times X \times X \rightarrow[0, \infty)$ is called a $D$-metric space, denoted by $(X, D)$ if $D$ satisfies

(i) $D(x, y, z)=0$ if and only if $x=y=z$ (coincidence),

(ii) $D(x, y, z)=D(p\{x, y, z\})$, where $p$ is a permutation of $x, y, z$ (symmetry),

(iii) $D(x, y, z) \leq D(x, y, a)+D(x, a, z)+D(a, y, z)$ for all $x, y, z, a \in X$ (tetrahedral inequality).

The nonnegative real function $D$ is called a $D$-metric on $X$. Some specific examples of $D$-metrics appear in [2]. A $D$-metric is a continuous function on $X^{3}$ in the topology of $D$-metric convergence, which is Hausdorff (see [5]).

In this paper, we establish two general fixed point principles for mappings in a $D$ metric space, which yield several fixed point theorems as corollaries.

2. Preliminaries. Let $f: X \rightarrow X$. The orbit of $f$ at the point $x \in X$ is the set $O(x)=$ $\left\{x, f x, f^{2} x, \ldots\right\}$. An orbit of $x$ is said to be bounded if there exists a constant $K>0$ such that $D(u, v, w) \leq K$ for all $u, v, w \in O(x)$. The constant $K$ is called a $D$-bound of $O(x)$. A $D$-metric space $X$ is said to be $f$-orbitally bounded if $O(x)$ is bounded for each $x \in X$. A sequence $x_{n} \subset X$ is said to be $D$-Cauchy if, for each $\varepsilon>0$, there exists a positive integer $n_{0}$ such that, for all $m>n, p \geq n_{0}, D\left(x_{m}, x_{n}, x_{p}\right)<\varepsilon$. A sequence $\left\{x_{n}\right\} \subset X$ is said to be $D$-convergent to a point $x \in X$ if, for each $\varepsilon>0$ there exists a positive integer $n_{0}$ such that, for all $m, n \geq n_{0}, D\left(x_{m}, x_{n}, x\right)<\varepsilon$. An orbit $O(x)$ is called $f$-orbitally complete if every $D$-Cauchy sequence in $O(x)$ converges to a point in $X$.

LEMMA 2.1 [4]. Let $\left\{x_{n}\right\} \subset X$ be a bounded sequence with $D$-bound $K$ satisfying

$$
D\left(x_{n}, x_{x+1}, x_{m}\right) \leq \lambda^{n} K
$$

for all positive integers $m>n$, and some $0 \leq \lambda<1$. Then $\left\{x_{n}\right\}$ is D-Cauchy. 


\section{Main results}

THEOREM 3.1. Let $(X, D)$ be a $D$-metric spaces, $f$ a selfmap of $X$. Suppose that there exists an $x_{0} \in X$ such that $O\left(x_{0}\right)$ is D-bounded and $f$-orbitally complete. Suppose also that $f$ satisfies

$$
D(f x, f y, f z) \leq \lambda \max \{D(x, y, z), D(x, f x, z)\} \quad \text { for } x, y, z \in \overline{O\left(x_{0}\right)}
$$

for some $0 \leq \lambda<1$. Then $f$ has a unique fixed point in $X$.

Proof. Suppose there exists an $n$ such that $x_{n}=x_{n+1}$. Then $f$ has $x_{n}$ as a fixed point in $X$. Therefore we may assume that all of the $x_{n}$ are distinct.

We wish to show that, for any positive integers $m, n, m>n$, that $D\left(x_{n+1}, x_{n+2}, x_{m}\right)$ $\leq \lambda^{n} K$, where $K$ is the $D$-bound of $O\left(x_{0}\right)$. The proof is by induction. From (3.1), for any $m$,

$$
D\left(x_{1}, x_{2}, x_{m}\right) \leq \lambda \max \left\{D\left(x_{0}, x_{1}, x_{m-1}\right), D\left(x_{0}, x_{1}, x_{m-1}\right)\right\} \leq \lambda K .
$$

Again using (3.1),

$$
D\left(x_{2}, x_{3}, x_{m}\right) \leq \lambda \max \left\{D\left(x_{2}, x_{3}, x_{m-1}\right), D\left(x_{1}, x_{2}, x_{m-1}\right)\right\} .
$$

Using (3.2),

$$
D\left(x_{2}, x_{3}, x_{m}\right) \leq \lambda \max \left\{D\left(x_{2}, x_{3}, x_{m-1}\right), \lambda K\right\} .
$$

Inequality (3.4) can be regarded as a recursion formula in $m$. Therefore

$$
D\left(x_{2}, x_{3}, x_{m}\right) \leq \lambda \max \left\{\lambda \max \left\{D\left(x_{2}, x_{3}, x_{m-2}\right), \lambda K\right\}, \lambda K\right\} \leq \lambda^{2} K .
$$

Assume the induction hypothesis. Then, from (3.1),

$$
\begin{aligned}
D\left(x_{n+1}, x_{n+2}, x_{m}\right) & \leq \lambda \max \left\{D\left(x_{n+1}, x_{n+2}, x_{m-1}\right), D\left(x_{n}, x_{n+1}, x_{m-1}\right)\right\} \\
& \leq \lambda \max \left\{D\left(x_{n+1}, x_{n+2}, x_{m-1}\right), \lambda^{n} K\right\} .
\end{aligned}
$$

Inequality (3.6) can be regarded as a recursion formula in $m$. Therefore,

$$
\begin{aligned}
D\left(x_{n+1}, x_{n+2}, x_{m}\right) & \leq \lambda \max \left\{\lambda \max \left\{D\left(x_{n+1}, x_{n+2}, x_{m-2}\right), \lambda^{n} K\right\}, \lambda^{n} K\right\} \\
& =\max \left\{\lambda^{2} D\left(x_{n+1}, x_{n+2}, x_{m-2}\right), \lambda^{n+2} K, \lambda^{n+1} K\right\} \\
& =\max \left\{\lambda^{2} D\left(x_{n+1}, x_{n+2}, x_{m-2}\right), \lambda^{n+1} K\right\} \\
& \leq \max \left\{\lambda^{2} \cdot \lambda \max \left\{D\left(x_{n+1}, x_{n+2}, x_{m-3}\right), \lambda^{n+1} K\right\}, \lambda^{n+1} K\right\} \\
& =\max \left\{\lambda^{3} D\left(x_{n+1}, x_{n+2}, x_{m-3}\right), \lambda^{n+1} K\right\} \leq \cdots \\
& \leq \max \left\{\lambda^{n} D\left(x_{n+1}, x_{n+2}, x_{m-n}\right), \lambda^{n+1} K\right\} \\
& \leq \max \left\{\lambda^{n} \cdot \lambda \max \left\{D\left(x_{n+1}, x_{n+2}, x_{m-n-1}\right), \lambda^{n+1} K\right\}, \lambda^{n+1} K\right\} \\
& =\lambda^{n+1} K,
\end{aligned}
$$

and $\left\{x_{n}\right\}$ is $D$-Cauchy by Lemma 2.1 . Since $X$ is $x_{0}$-orbitally complete, there exists a $p \in X$ with $\lim x_{n}=p$. 
In (3.1) set $x=x_{n}, z=p$ to obtain

$$
D\left(x_{n+1}, x_{n+1}, f p\right) \leq \lambda \max \left\{D\left(x_{n}, x_{n}, p\right), D\left(x_{n}, x_{n+1}, p\right)\right\} .
$$

Taking the limit of (3.8) as $n \rightarrow \infty$ yields $D(p, p, f p) \leq \lambda D(p, p, p)=0$, and $p=f p$. To prove uniqueness, suppose that $q$ is also a fixed point of $f$. Then, from (3.1),

$$
D(p, p, q)=D(f p, f p, f q) \leq \lambda \max \{D(p, p, q), D(p, f p, q)\}=\lambda D(p, p, q),
$$

which implies that $p=q$.

COROLlary 3.2 [2, Theorem 2.1]. Let $f$ be a selfmap of a complete and bounded $D$-metric space $X$ satisfying

$$
D(f x, f y, f z) \leq \lambda D(x, y, z)
$$

for all $x, y, z \in X$, for some $0 \leq \lambda<1$. Then $f$ has a unique fixed point $p$, and $f$ is continuous at $p$.

Proof. In (3.10) set $y=f x$ to obtain (3.1). Then, from Theorem 3.1, $f$ has a unique fixed point $p$.

To prove continuity, let $\left\{z_{n}\right\} \subset X$ with $\lim z_{n}=p$. From (3.10),

$$
D\left(p, p, f z_{n}\right)=D\left(f p, f p, f z_{n}\right) \leq \lambda D\left(p, p, z_{n}\right) .
$$

Taking the limit as $n \rightarrow \infty$ gives $\lim \sup D\left(p, p, f z_{n}\right)=0$, and $\liminf D\left(p, p, f z_{n}\right)=0$ which implies that $\lim f z_{n}=p=f p$, and $f$ is continuous at $p$.

COROLlary 3.3 [2, Corollary 1.1]. Let $f$ be a selfmap of a complete and bounded $D$-metric space satisfying the condition that there exists a positive integer $q$ such that

$$
D\left(f^{q} x, f^{q} y, f^{q} z\right) \leq \lambda D(x, y, z)
$$

for all $x, y, z \in X$, for some $0 \leq \lambda<1$. Then $f$ has a unique fixed point $p$, and $f$ is $f$-orbitally continuous at $p$.

Proof. Define $T=f^{q}$. Then (3.12) reduces to (3.10), and $T$ has a unique fixed point $p$ by Corollary 3.2; i.e., $p=T p=f^{q} p$. Thus $f p=f^{q+1} p=T(f p)$, and $f p$ is also a fixed point of $T$. Uniqueness implies that $f p=p$, and $p$ is a fixed point of $f$. Condition (3.12) implies the uniqueness of $p$ as a fixed point of $f$.

For the continuity, let $\left\{z_{n}\right\} \subset O(f)$, with $\lim z_{n}=p$. From (3.12),

$$
D\left(f^{q} p, f^{q} p, f^{q} z_{n}\right) \leq \lambda D\left(p, p, z_{n}\right) .
$$

Taking the limit as $n \rightarrow \infty$ shows that $\lim f^{q} z_{n}=p=f^{a} p$, and $f^{q}$ is $f$-orbitally continuous at $p$. But, since each $z_{n} \in O(f), \lim f^{q} z_{n}=\lim f z_{n+q-1}$, and $f$ is $f$-orbitally continuous at $p$.

COROLLARY 3.4. Let $f$ be a selfmap of $X, X$ an $f$-orbitally bounded and complete D-metric space satisfying

$$
D(f x, f y, f z) \leq \alpha\left[\frac{1+D(x, f x, z)}{1+D(x, y, z)}\right] D(y, f y, z)+\beta D(x, y, z)
$$


for all $x, y, z \in X, \alpha, \beta \geq 0, \alpha+\beta<1$. Then $f$ has a unique fixed point $p$ and $f$ is continuous at $p$.

Proof. In (3.14) set $y=f x$ to obtain

$$
\begin{aligned}
D\left(f x, f^{2} x, f z\right) & \leq \alpha D\left(f x, f^{2} x, z\right)+\beta D(x, f x, z) \\
& \leq \lambda \max \left\{D\left(f x, f^{2} x, z\right), D(x, f x, z)\right\},
\end{aligned}
$$

where $\lambda=\alpha+\beta<1$, and (3.1) is satisfied. The conclusion follows from Theorem 3.1.

To prove the continuity of $f$ at $p$, let $\left\{z_{n}\right\} \subset X$ with $\lim z_{n}=p$. In (3.14) set $x=z$ $=p, y=z_{n}$, to obtain

$$
\begin{aligned}
D\left(p, f z_{n}, p\right) & =D\left(f p, f z_{n}, f p\right) \\
& \leq \alpha\left[\frac{1+D(p, f p, p)}{1+D\left(p, z_{n}, p\right)}\right] D\left(z_{n}, f z_{n}, p\right)+\beta D\left(p, z_{n}, p\right) \\
& \leq \alpha D\left(z_{n}, f z_{n}, p\right)+\beta D\left(p, z_{n}, p\right) .
\end{aligned}
$$

Taking the limsup of both sides of (3.16) as $n \rightarrow \infty$ yields

$$
D\left(p, \lim \sup f z_{n}, p\right) \leq \alpha D\left(p, \lim \sup f z_{n}, p\right),
$$

which implies that $\lim \sup f z_{n}=p$. Similarly, taking the liminf of both sides of (3.16) as $n \rightarrow \infty$ yields

$$
D\left(p, \liminf f z_{n}, p\right) \leq \alpha D\left(p, \liminf f z_{n}, p\right),
$$

which implies that $\liminf f z_{n}=p$. Therefore $\lim f z_{n}=p=f p$, and $f$ is continuous at $p$.

COROLLARY 3.5. Let $f$ be a selfmap of an $f$-orbitally bounded and complete $D$ metric space $X, q$ a fixed positive integer. Suppose that $f$ satisfies

$$
D\left(f^{q} x, f^{q} y, f^{q} z\right) \leq \alpha\left[\frac{1+D\left(x, f^{q} x, z\right)}{1+D(x, y, z)}\right] D\left(y, f^{q} y, z\right)+\beta D(x, y, z)
$$

for all $x, y, z \in X$, where $\alpha, \beta \geq 0, \alpha+\beta<1$. Then $f$ has a unique fixed point $p$ and $f$ is $f$-orbitally continuous at $p$.

Proof. Set $T=f^{q}$. Then $T$ satisfies (3.14). Therefore $T$ has a unique fixed point at $p$, and is continuous at $p$. A standard argument then verifies that $f$ has $p$ as a unique fixed point. As in the proof of Corollary $3.3, f$ is $f$-orbitally continuous at $p$.

4. $\alpha$-condensing maps. For any set $A$ in a $D$-metric space $X$, the $D$-diameter of $A, \delta(A)$, is defined by $\delta(A)=\sup _{x, y, z \in A} D(x, y, z)$. The measure of noncompactness of a bounded set $A$ in a $D$-metric space $X$ is a nonnegative real number $\alpha(A)$ defined by

$$
\alpha(A)=\inf \left\{\gamma>0: A=\cup_{i=1}^{n}: A_{i} \text { for which } \delta\left(A_{i}\right) \leq \gamma \text { for } i=1,2, \ldots, n\right\} .
$$


DEFinition 4.1. A selfmap $f$ of $X$ is called $\alpha$-condensing if, for any bounded set $A$ in $X, f(A)$ is bounded and $\alpha(f(A))<\alpha(A)$ if $\alpha(A)>0$.

Some authors refer to $\alpha$-condensing maps as densifying maps.

LEMMA 4.2. Let $f: X \rightarrow X, X$ an $f$-orbitally bounded and complete D-metric space, be $\alpha$-condensing. Then $\overline{O(x)}$ is compact for each $x \in X$.

Proof. Let $x \in X$ and define $A \subset X$ by $A=\left\{x_{n}\right\}$, where $x_{n}=f^{n} x$. Then

$$
A=\left\{x, f x, f^{2} x, \ldots\right\}=\{x\} \cup\left\{f x, f^{2} x, \ldots\right\}=\{x\} \cup f(A) .
$$

Therefore, if $A$ is not precompact, then $\alpha(A)=\alpha(f(A))<\alpha(A)$, a contradiction. Therefore $\bar{A}=\overline{O(x)}$ is compact, since $\bar{A}$ is a complete $D$-metric space.

Define $\delta(x, y, z)=\delta(O(x) \cup O(y) O(z))$

THEOREM 4.3. Let $f$ be a continuous compact selfmap of a bounded D-metric space $X$, satisfying

$$
D\left(f^{r} x, f^{s} y, f^{t} z\right)<\delta(x, y, z) \text { for each } x, y, z \in X \text {, with two of }\{x, y, z\} \text { distinct, }
$$

where $r, s$, and $t$ are fixed positive integers. Then $f$ has a unique fixed point in $X$.

Proof. Since $f$ is compact, there exists a compact subset $Y$ of $X$ containing $f X$. Then $f Y \subset Y$ and $A:=\cap_{n=1}^{\infty} f^{n} Y$ is a nonempty compact $f$-invariant subset of $X$ which is mapped by $f$ onto itself. $A$ has the same properties with respect to $f^{r}, f^{s}$, and $f^{t}$.

Suppose that $\delta(A)>0$. Since $A$ is compact there exist $x, y, z \in A$ such that $\delta(A)=$ $D(x, y, z)$. Since $f A=A$, there exist $x^{\prime}, y^{\prime}$, and $z^{\prime}$ in $A$ such that $x=f^{r} x^{\prime}, y=f^{s} y^{\prime}$, and $z=f^{t} z^{\prime}$. Then, from (4.3),

$$
\delta(A)=D(x, y, z)=D\left(f^{r} x^{\prime}, f^{s} y^{\prime}, f^{t} z^{\prime}\right)<\delta(x, y, z)=\delta(A),
$$

a contradiction. Therefore $A$ consists of a single point, which is a fixed point of $f$.

Suppose $p$ and $q$ are fixed points of $f, p \neq q$. Then, from (4.3),

$$
0<D(p, p, q)=D\left(f^{r} p, f^{s} p, f^{t} q\right)<D(p, p, q),
$$

a contradiction. Therefore the fixed point is unique.

COROLlary 4.4 [8, Theorem 2]. Let $X$ be a compact $D$-metric space, $f$ a continuous selfmap of $X$ satisfying

$$
\begin{aligned}
& D(f x, f y, f z) \\
& \quad<\max \{D(x, y, z), D(x, f x, z), D(y, f y, z), D(x, f y, z), D(y, f x, z)\} D(p, p, q)
\end{aligned}
$$

for all $x, y, z \in X$ with $x \neq f x, y \neq f y$, or $z \neq f z$. Then $f$ has a unique fixed point $p$ in $X$. 
Proof. Inequality (4.6) implies that $D(f x, f y, f z)<\delta(x, y, z)$, and the existence and uniqueness of a fixed point $p$ follows from Theorem 4.3.

For continuity, let $\left\{z_{n}\right\} \subset X$ with $z_{n} \neq p$ for each $n$ and $\lim z_{n}=p$. From (4.6)

$$
D\left(p, p, f z_{n}\right)=D\left(f p, f p, f z_{n}\right)<D\left(p, f p, z_{n}\right) .
$$

Taking the limit as $n \rightarrow \infty$ implies that $f$ is continuous at $p$.

THEOREM 4.5. Let $f$ be an $f$-orbitally continuous $\alpha$-condensing selfmap of a complete bounded $D$-metric space X. Let $a \in X$. If (4.3) holds on $\overline{O(a)}$, then $f$ has a unique fixed point $p \in \overline{O(a)}$, and $\lim _{n} f^{n} x=p$ for each $x \in \overline{O(a)}$.

Proof. From Lemma 4.2, $\overline{O(a)}$ is compact. Since $f$ is a continuous $\alpha$-condensing selfmap of $\overline{O(a)}, f$ is compact. Now apply Theorem 4.3.

COROLLARY 4.6. Let $f$ be a continuous $\alpha$-condensing selfmap of a complete bounded $D$-metric space $X$ satisfying (4.6) for all $x, y, z \in X$ with $x \neq f x, y \neq f y$, or $z \neq f z$. Then $f$ has a unique fixed point $p$ in $X$.

As in the proof of Corollary $4.4, D(f x, f y, f z)<\delta(x, y, z)$ and the result follows from Theorem 4.5 .

THEOREM 4.7. Let $f$ be a selfmap of a $D$-metric space $X$. Suppose that there exists a point $a \in X$ with $\overline{O(a)}$ bounded and complete. Suppose that $f$ is continuous and $\alpha$-condensing on $\overline{O(a)}$ and satisfies (4.3) for each $x, y, z \in \overline{O(a)}$ with two of $\{x, y, z\}$ distinct, and $x \neq f x, y \neq f y, z \neq f z$. Then $f$ has a fixed point in $\overline{O(a)}$.

Proof. By Lemma $4.2 \overline{O(a)}$ is compact. If there exists some integer $n$ for which $f^{n} a=f^{n+1} a$, then $f$ has a fixed point in $\overline{O(a)}$. Assume that $f^{n} a \neq f^{n+1} a$ for each $n$. Note that $f$, restricted to $\overline{O(a)}$ is a continuous compact selfmap of $\overline{O(a)}$. Suppose that $u \neq f u$ for each cluster point $u$ of $\overline{O(a)}$. Then $f$ satisfies condition (4.3) for all $x, y, z \in \overline{O(a)}$, with two of $\{x, y, z\}$ distinct. Therefore, by Theorem $4.3, f$, restricted to $\overline{O(a)}$, has a unique fixed point $p \in \overline{O(a)}$. This contradicts the assumption that $u \neq f u$ for each cluster point $u$ of $\overline{O(a)}$. Therefore $u=f u$ for some cluster point $u \in \overline{O(a)}$.

The proofs of Theorems 4.3, 4.5, and 4.7 are very similar to their metric space counterparts in [6] and [7], but have been given here for completeness.

The following results are proved using the proof technique analogous to the corresponding metric space theorems.

THEOREM 4.8. Let $f$ be a selfmap of $X$, an $f$-orbitally bounded and complete $D$ metric space. Suppose that $f$ is $\alpha$-condensing, $f$-orbitally continuous and satisfies

$$
D(f x, f y, f z)<\alpha\left[\frac{1+D(x, f x, z)}{1+D(x, y, z)}\right] D(y, f y, z)+\beta D(x, y, z)=M(x, y, z)
$$

for all $x, y, z \in X$ with $x \neq f x, y \neq f y, z \neq f z$, where $\alpha, \beta>0, \alpha+\beta \leq 1$. Then $f$ has a unique fixed point $p \in X$ and $f$ is continuous at $p$.

Proof. If $\alpha+\beta<1$, the result follows from Corollary 3.4. Therefore we assume that $\alpha+\beta=1$. Let $x_{0} \in X$ and define $x_{n+1}=f x_{n}, n \geq 0$. From Lemma 4.2 it follows that $\overline{O\left(x_{0}\right)}$ is compact. Obviously $f: \overline{O\left(x_{0}\right)} \rightarrow \overline{O\left(x_{0}\right)}$. 
CASE I. There exists some $x, y, z \in \overline{O\left(x_{0}\right)}$ for which $M=0$. Then $y=f y=z=x$, and $y$ is a fixed point of $f$. Inequality (4.8) implies uniqueness.

CASE II. $M \neq 0$ for all $x, y, z \in \overline{O\left(x_{0}\right)}$. Define a function $F:\left(\overline{O\left(x_{0}\right)}\right)^{3} \rightarrow[0, \infty)$ by

$$
F(x, y, z)=\frac{D(f x, f y, f z)}{M(x, y, z)} .
$$

The function $F$ is well defined on $\left(\overline{O\left(x_{0}\right)}\right)^{3}$ since $M \neq 0$ on $\overline{O\left(x_{0}\right)}$.

Since $F$ is continuous on $\overline{O\left(x_{0}\right)}$, it attains its maximum value at some point $(u, v, w)$ $\in \overline{O\left(x_{0}\right)}$. We call this maximum value $c$. From (4.8) it follows that $0<c<1$. Therefore

$$
\begin{aligned}
D(f x, f y, f z) & \leq c M(x, y, z) \\
& \leq \alpha^{\prime}\left[\frac{1+D(x, f x, z)}{1+D(x, y, z)}\right] D(y, f y, z)+\beta^{\prime} D(x, y, z)
\end{aligned}
$$

for all $x, y, z \in \overline{O\left(x_{0}\right)}$, where $\alpha^{\prime}=c \alpha>0, \beta^{\prime}=c \beta>0$, and $\alpha^{\prime}+\beta^{\prime}=c(\alpha+\beta)<1$. Since $\overline{O\left(x_{0}\right)}$ is compact, it is bounded and complete. The result follows from Corollary 3.4.

COROLLARY 4.9. Let $f$ be a selfmap of a complete and $f$-orbitally bounded $D$-metric space. Suppose that $f$ is $\alpha$-condensing and $f$-orbitally continuous. Let $q$ be a positive integer. Suppose that $f$ satisfies

$$
D\left(f^{q} x, f^{q} y, f^{q} z\right)<\alpha\left[\frac{1+D\left(x, f^{q} x, z\right)}{1+D(x, y, z)}\right] D\left(y, f^{q} y, z\right)+\beta D(x, y, z)
$$

for all $x, y, z \in X$ for which the right-hand side of (4.11) is not zero, where $\alpha, \beta>0$, $\alpha+\beta \leq 1$. Then $f$ has a unique fixed point $p$ and $f$ is $f$-orbitally continuous at $p$.

Proof. Set $T=f^{q}$. Then $T$ satisfies (4.8), and the existence and uniqueness of the fixed point $p$, for $T$, follows from Theorem 4.8. It then follows that $p$ is the unique fixed point for $f$. The continuity argument is the same as that used in the proof of Corollary 3.3.

COROLLARY 4.10. Let $f$ be a continuous selfmap of a compact D-metric space satisfying (4.8). Then $f$ has a unique fixed point $p$, and $f$ is continuous at $p$.

This result is an immediate consequence of Theorem 4.8 .

Corollary 4.10 includes [3, Theorem 2.2] as a special case.

\section{REFERENCES}

[1] B. C. Dhage, A study of some fixed point theorems, Ph.D. thesis, Marathwada University, Aurangabad, India, 1984.

[2] _ Generalised metric spaces and mappings with fixed point, Bull. Calcutta Math. Soc. 84 (1992), no. 4, 329-336. MR 93k:54075. Zbl 782.54037.

[3] _ On continuity of mappings in D-metric spaces, Bull. Calcutta Math. Soc. 86 (1994), no. 6, 503-508. CMP 1352 051. Zbl 836.54006.

[4] _ On Kanan type maps in D-metric spaces, J. Natur. Phys. Sci. 11 (1997), 21-38. CMP 1659334.

[5] _ Generalized metric spaces and topological structure, I, An. Ştiinț. Univ. Al. I. Cuza Iaşi. Mat. (N.S.) (1998), to appear. 
[6] S. Park, On studying maps of metric spaces, Honam J. 1 (1981), 23-30.

[7] S. Park and B. E. Rhoades, Extensions of some fixed point theorems of Fisher and Janos, Bull. Acad. Polon. Sci. Sér. Sci. Math. 30 (1982), no. 3-4, 167-169. MR 83j:54046. Zbl 503.54050.

[8] B. E. Rhoades, A fixed point theorem for generalized metric spaces, Internat. J. Math. Math. Sci. 19 (1996), no. 3, 457-460. CMP 1386 544. Zbl 857.54044.

Dhage and Pathan: Mathematical Research Centre, Mahatma Gandhi MahavidyALAYA, AHMEDPUR-413 515, INDIA

RHOADES: DePARTMENT OF MATHEMATICS, INDIANA UNIVERSITY, BLOOMINGTON, INDIANA 47405-4301, USA

E-mail address: rhoades@indiana.edu 


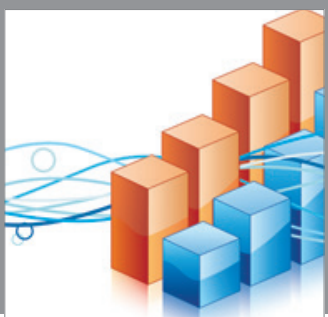

Advances in

Operations Research

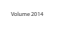

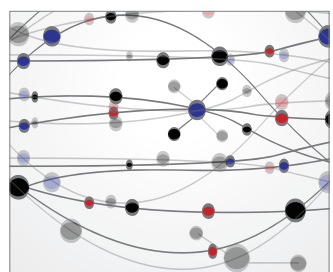

\section{The Scientific} World Journal
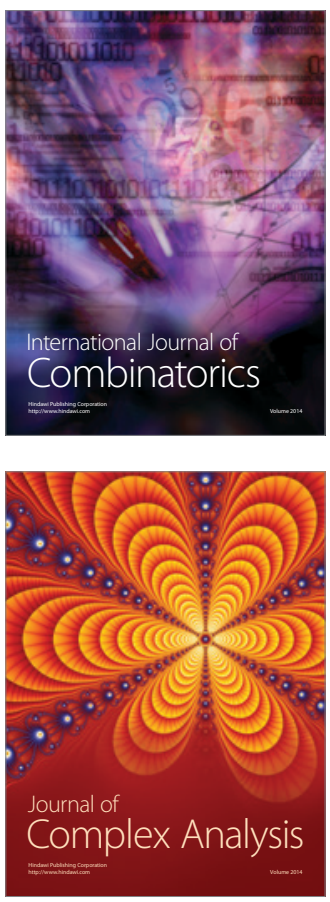

International Journal of

Mathematics and

Mathematical

Sciences
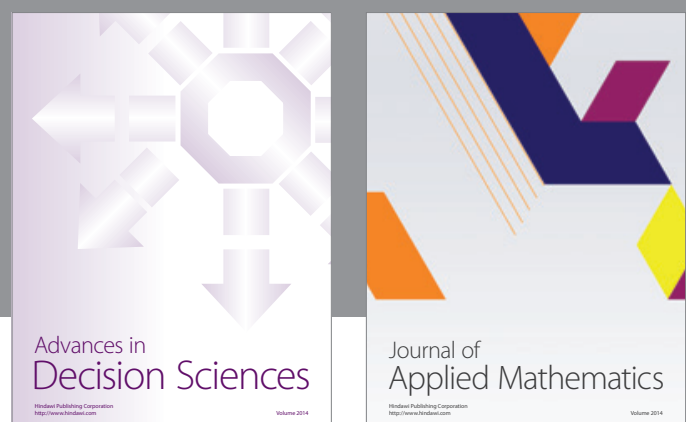

Journal of

Applied Mathematics
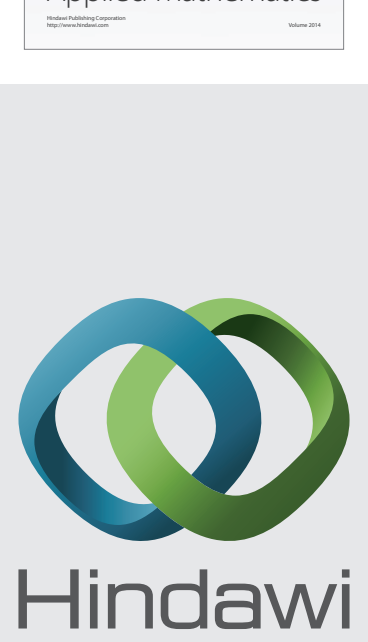

Submit your manuscripts at http://www.hindawi.com
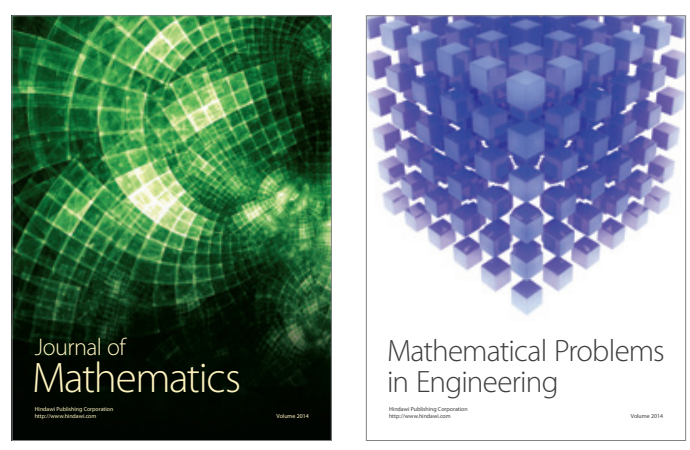

Mathematical Problems in Engineering
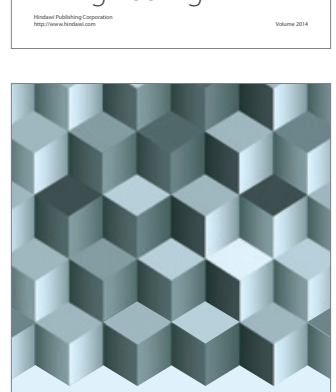

Journal of

Function Spaces
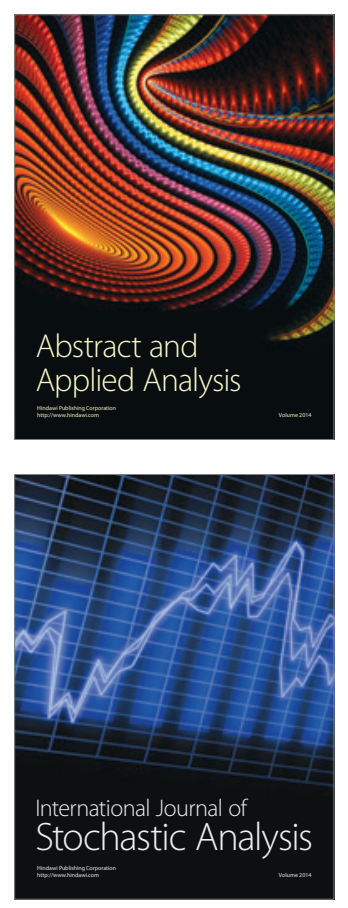

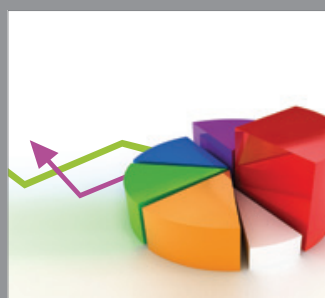

ournal of

Probability and Statistics

Promensencen
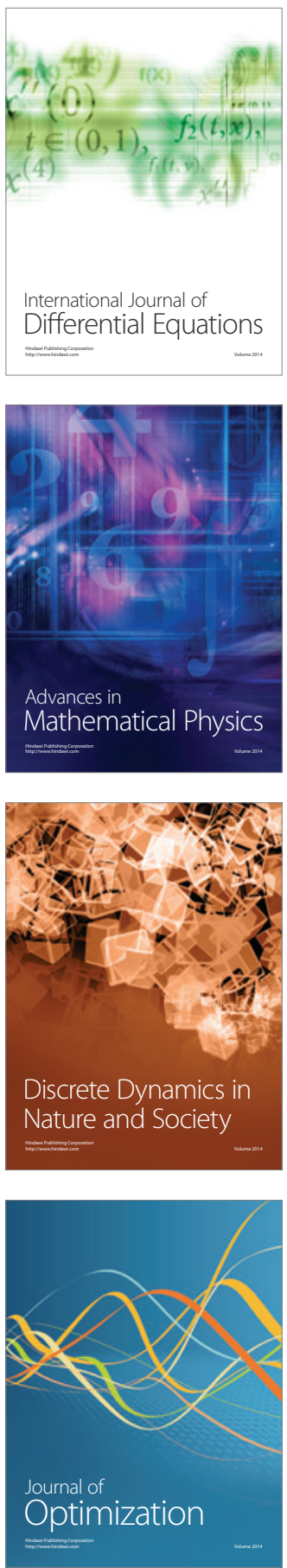